\title{
A Stable Multipath QoS Routing Protocol Based on the Remaining Power of the Nodes in Opportunistic Networks
}

\author{
Xuebin Ma \\ Department of Computer Sciences \\ Inner Mongolia University \\ Hohhot, China \\ csmaxuebin@imu.edu.cn
}

\author{
Liting Wang \\ Department of Computer Sciences \\ Inner Mongolia University \\ Hohhot, China \\ wanglt99@gmail.com
}

\begin{abstract}
With the growing popularity of smart devices, people have increasingly requirements for network's quality of service (QoS). There is a growing tendency in opportunistic networks to establish stable paths by using long path lifetimes, low routing overhead and high packet delivery ratio. According to recent analytical results, the lifetime of a path depends on the length of the path, the lifetime of each link and remaining power of nodes in the path. Meanwhile, the lifetime of a path can reflect the path stability. This paper presents a backup routing protocol based on the stable nodes (SNBR), it achieves high path stability by path length, link lifetime and remaining power of nodes. In SNBR, the primary path composes of stable nodes and intermediate nodes, and the stable nodes are determined by the remaining power of the nodes. In addition, every link of the backup path consists of disjoint sub-paths between two adjacent and stable nodes. Analytical results indicate that SNBR has good performance in terms of path lifetime and packet delivery ratio, and thus improves the QoS of the network.
\end{abstract}

Keywords-opportunistic networks; stability; multipath routing; QoS

\section{INTRODUCTION}

An opportunistic network is such a kind of self-organized network that does not necessarily have a full path between source and destination. It utilizes encounter chance of mobile nodes to realize ad hoc network communication [1]. Delay tolerant network (DTN) is first proposed by tolerant network research group for communication of interplanetary network (IPN), and its main objective is to support inter-connection of heterogeneous network which has intermittent connectivity, large delay and high error rate, such as Internet and sensor network, mobile ad hoc network (MANET) [2]. Thus, the opportunistic network can be treated as a wireless ad hoc network with the general characteristics of the DTN network.

When messages are transmitted between nodes, the routing protocols exist at least a complete end-to-end path in the traditional MANET [2, 3]. At some point, MANET network may be divided into unconnected sub-regions. When source node and destination node are in different domains, routing protocols in MANET cannot find the path to the destination node. However, this does not represent that nodes cannot communicate each other without a complete path from source to destination. Since the mobility of node is random, two nodes can exchange data in a hop-by-hop method.

With the further research and extensive application of MANET and wireless sensor network (WSN), the opportunistic networks can deny and change the routing model of the traditional networks. In opportunistic networks, Epidemic routing protocol, Spray and Wait routing protocols and code-based routing protocols have lower latency, but they require huge network overhead. Therefore, the QoS of the network has not been guaranteed.

In order to improve stability of path under QoS constraints, this paper presents a backup routing protocol based on the stable nodes (SNBR). In SNBR, the primary path and backup path are constructed by stable nodes and lifetime of every link. When the primary path fails, the appropriate backup path can be utilized. Through theoretical analysis, it turns out SNBR protocol enhances lifetime of the path, thereby improving stability of the path and guaranteeing the QoS of the network.

The rest of this paper is organized as follows: Section II presents some related works. Section III describes the SNBR protocol in detail. Section IV discusses the performance of the SNBR protocol by theoretical analysis. Finally, in Section V we conclude with the performance of SNBR.

\section{RELATED WORK}

They have a certain similarity to both opportunistic networks and mobile ad hoc network (MANET) that have no fixed infrastructure. However, topology of the network dynamically changes which can lead to the link frequently interrupt, and thus it is hard to establish a stable path in opportunistic networks. In recent years, in order to improve stability of the paths in MANET, scholars have developed a variety of routing protocols. C. E. Perkins and E. M. Royer presented AODV [4]. In AODV, when one route is needed for communication, the source node starts the route discovery process, and keeps maintenance on the active node and exchanges routing table periodically, which greatly reduces the cost of routing information. Marina and Das proposed an ad hoc on-demand multipath distance vector routing (AOMDV) protocol which is an extension of AODV [5]. It is used to calculate loop-free paths and disjoint link. Loop-free paths are determined through the use of broadcasting hops, and the link-disjointness-based multipath is obtained through a particular flooding strategy. Xuefei Li and Laurie Cuthbert proposed a node-disjointness-based 
multipath QoS routing protocol (NDMR) which alters and extends the AODV [6]. It enables accumulation feature of DSR protocol by using the request / response packet, and also finds multiple and disjoint paths through a low routing overhead. Song Guo, Oliver Yang and Yantai Shu proposed a backup source routing protocol (BSR), when the primary path is interrupted, it is used to establish and maintain a backup path to reduce the frequency of route discovery flooding [7].

Mohammed Tarique, Kemal E. Tepe, Sasan Adibi and Shervin Erfani conducted a research on MANET multipath routing protocol [8]. The main goal of multipath routing protocol is to establish reliable communication to ensure load balance and improve QoS. P. Rama Devi and D. Srinivasa Rao proposed a QoS enhanced hybrid multipath routing protocol which has a proactive topology discovery and reactive path discovery, thereby reducing the routing overhead [9]. In proactive topology discovery phase, each node collected battery power, queue length, and the remaining bandwidth of other nodes and stored them in topology information table (TIT). Topology is discovered through the exchange of TIT between nodes. When the source forwards the data packet to the destination, reactive discovery technology is used to create multipath routing.

LAURA MARIE FEENEY proposed a model for assessing the energy behavior of MANET network, and used this model to test energy consumption of two famous MANET routing protocols (DSR and AODV) [10]. Nils Aschenbruck and Matthias Schwamborn proposed a mobility model in a real scene to assess the controllability of the wireless network which could measure mobile traces of opportunistic networks [11].

$\mathrm{n}$ conclusion, in MANET network, a variety of routing protocols were proposed to improve the stability of the path, such as multi-path routing protocols, QoS routing protocol. But in opportunistic networks, currently, there is no corresponding multipath routing protocol is proposed. Therefore, this paper proposes a backup routing protocol based on the stable nodes (SNBR), which is a kind of multipath routing protocol satisfies the QoS constraints. The SNBR protocol enhances stability of the path and improves QoS of the network.

\section{SNBR Routing Protocol}

We first analyze activities of the nodes in the research scene and determine the initial position coordinates of each node by global positioning system (GPS). Then, position coordinates of the nodes can be obtained by the mobility model since the nodes move directionally. Secondly, the stable nodes are selected by remaining power of the nodes, and the common nodes in both the primary path and backup path are replaced with the stable nodes. Finally, we can achieve the expiration time of the entire path by calculating the expiration time of each link.

\section{A. Path Discovery Process}

1) Activity behaviors of the mobile nodes: We observe and analyze mobile behaviors through students in the universities as well as workers in the companies. We find that the activities of the mobile nodes have a certain regularity and directivity. We collects the initial position coordinates of nodes by global positioning system (GPS) and periodically broadcasts request packet to obtain the position coordinates of other nodes. According to both moving direction and speed of the nodes, we can achieve new position coordinates of the mobile nodes at a time.

2) Selection process of the stable nodes: We select the optimal path from source node $\mathrm{S}$ and destination node $\mathrm{D}$ by both the remaining power of the nodes and the lifetime of the paths. The selection process of the stable nodes in the path is shown in Figure 1. The selection process of the stable nodes in Figure 1 is described as follow.

a) The initial position coordinate of a node is determined by GPS, It can judge whether it is in the research scene: If the node is in the scene, we will continue to execute the step (b); otherwise, ends the operation .

b) To determine whether the node has been never visited: If the current node is not visited, then we mark the node to distinguish from other non-visited nodes and record the current remaining power of the node, and we will continue to execute the step (c); otherwise, returns to the step (a).

c) To determine whether the remaining power of the tagged node is in high power range: If the node is in the high power range, it will be chose as stable node; otherwise, returns to the step (a).

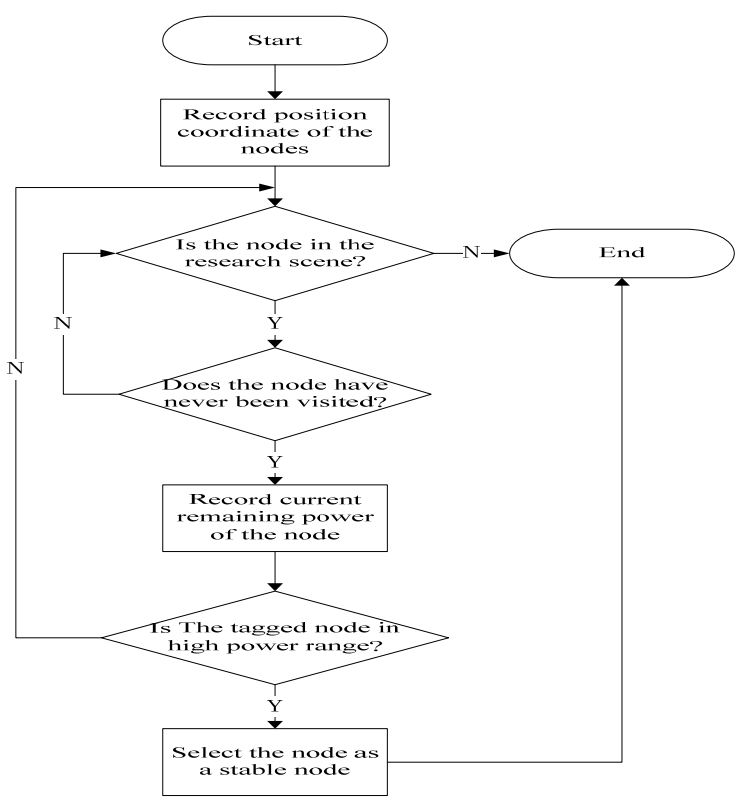

Figure 1. Selection process of the stable nodes

3) Selection criteria of the stable nodes: In order to improve lifetime of the path, we select relatively stable nodes to maintain the path. The stable nodes are selected by remaining power of the nodes. Both the primary path and the backup path have common nodes, the common nodes compose of the stable nodes. 
a) To estimate remaining power of the nodes: At time $t$, the remaining power of a node $(P(t))$ is given by Eq. 1 . Description of the corresponding parameters is shown in Table 1.

$$
P(t)=P(t-1)-M^{*} P_{t r}(t-1, t)-N^{*} P_{r e}(t-1, t)-P_{\text {iit }}(t-1, t) .
$$

TABLE I. DESCRIPTION OF THE RELEVANT PARAMETERS

\begin{tabular}{|c|c|}
\hline$P(t)$ & remaining power of the node at time $t$ \\
\hline$P(t-1)$ & remaining power of the node at time $t-1$ \\
\hline$P_{t r}(t-1, t)$ & $\begin{array}{l}\text { the consumed power for sending a data } \\
\text { packet from time } t-1 \text { to } t\end{array}$ \\
\hline$P_{r e}(t-1, t)$ & $\begin{array}{l}\text { the consumed power for receiving a data } \\
\text { packet from time } t-1 \text { to } t\end{array}$ \\
\hline$P_{\text {int }}(t-1, t)$ & $\begin{array}{l}\text { the consumed power of the node from } \\
\text { time } t-1 \text { to } t\end{array}$ \\
\hline$N$ & $\begin{array}{c}\text { The number of transmitted packet from } \\
\text { the time } t-1 \text { to } t\end{array}$ \\
\hline$M$ & $\begin{array}{l}\text { The number of received packet from the } \\
\text { time } t-1 \text { to } t\end{array}$ \\
\hline
\end{tabular}

b) To select the range of the stable nodes: Let $P_{t h}(t)$ be the threshold of remaining power, and remaining power of the nodes is divided into two ranges: low power range and high power range.

- When $P(t) \geq P_{t h}(t)$, indicating that remaining power of the node is in the high power range;

- When $P(t)<P_{t h}(t)$, indicating that remaining power of the node is in the low power range.

\section{B. Path Maintenance}

1) The mobility model of nodes [12]: The initial positions of nodes $i$ and $j$ have coordinates values $\left(X_{i}, Y_{i}\right)$ and $\left(X_{j}, Y_{j}\right)$, respectively. Nodes $i$ and $j$ move with variable velocities in a particular direction. Assumed that the nodes move in the positive $\mathrm{x}$-axis direction, the angles of nodes $i$ and $j$ are $\alpha$ and $\beta$, respectively. At time $t$, the moved distances of nodes $i$ and $j$ are $d 1$ and $d 2$, and their new coordinates values are $\left(X_{i}^{\prime}, Y_{i}^{\prime}\right)$ and $\left(X_{j}^{\prime}, Y_{j}^{\prime}\right)$, respectively as shown in Figure 2.

At time $\mathrm{T}=0$, the Euclidean distance of nodes $i$ and $j$ is at positions $\left(X_{i}, Y_{i}\right)$ and $\left(X_{j}, Y_{j}\right)$, as given in Eq. 2.

$$
d_{(i j, 0)}=\sqrt{\left|X_{i}-X_{j}\right|^{2}+\left|Y_{i}-Y_{j}\right|^{2}} .
$$

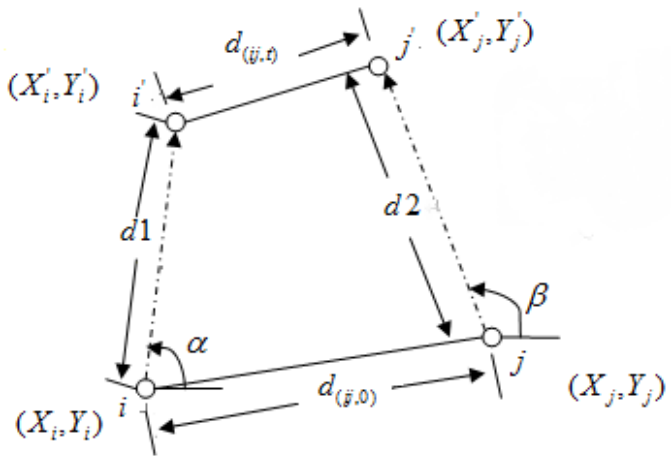

Figure 2. The distance of mobile nodes: before mobility and after mobility

In realistic scenario, the nodes move with a variable speed in a particular duration. It is necessary to account the speed of nodes at the beginning and end of mobility. Let $V_{i}^{s}, V_{i}^{f}, V_{j}^{s}$ and $V_{j}^{f}$ be the initial and final velocities of nodes $i$ and $j$, respectively. The distances $d 1$ and $d 2$ moved by two nodes at time $\mathrm{T}=t$ are given by Eqs. 3 and 4 , respectively.

$$
\begin{aligned}
& d 1=\frac{1}{2}\left(V_{i}^{s}+V_{i}^{f}\right) \times t . \\
& d 2=\frac{1}{2}\left(V_{j}^{s}+V_{j}^{f}\right) \times t .
\end{aligned}
$$

At time $\mathrm{T}=t$, node $i$ has a new location $\left(X_{i}^{\prime}, Y_{i}^{\prime}\right)$ when it moves a distance d 1 in direction $\alpha$. It is shown in Figure 3 . The values $X_{i}^{\prime}$ and $Y_{i}^{\prime}$ are given by Eqs.5 and 6, respectively.

$$
\begin{gathered}
X_{i}^{\prime}=X_{i}+d 1 \operatorname{Cos} \alpha=X_{i}+\frac{1}{2}\left(V_{i}^{s}+V_{i}^{f}\right) \times t \operatorname{Cos} \alpha . \\
Y_{i}^{\prime}=Y_{i}+d 1 \operatorname{Sin} \alpha=Y_{i}+\frac{1}{2}\left(V_{i}^{s}+V_{i}^{f}\right) \times t \operatorname{Sin} \alpha .
\end{gathered}
$$

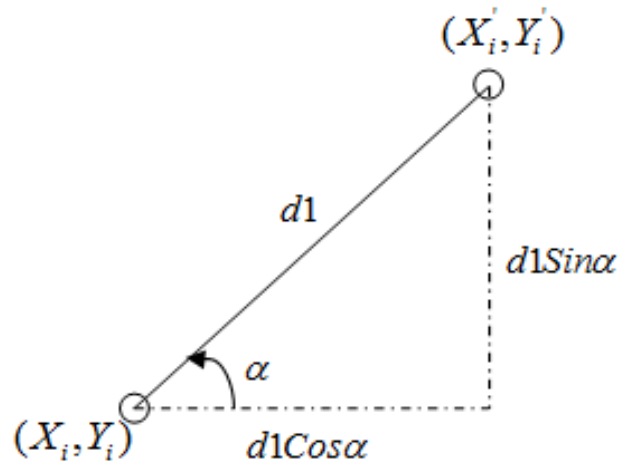

Figure 3. The new position coordinates of the mobile nodes after mobility

Similarly, node $j$ has a new location $\left(X_{j}^{\prime}, Y_{j}^{\prime}\right)$ when it moves a distance $d 2$ in direction $\beta$. The coordinate values of new position and are given by Eqs. 7 and 8 , respectively. 


$$
\begin{gathered}
X_{j}^{\prime}=X_{j}+d 2 \operatorname{Cos} \beta=X_{j}+\frac{1}{2}\left(V_{j}^{s}+V_{j}^{f}\right) \times t \operatorname{Cos} \beta . \\
Y_{j}^{\prime}=Y_{j}+d 2 \operatorname{Sin} \beta=Y_{j}+\frac{1}{2}\left(V_{j}^{s}+V_{j}^{f}\right) \times t \operatorname{Sin} \beta .
\end{gathered}
$$

At time $\mathrm{T}=t$, nodes $i$ and $j$ move to new positions $\left(X_{i}^{\prime}, Y_{i}^{\prime}\right)$ and $\left(X_{j}^{\prime}, Y_{j}^{\prime}\right)$, respectively, and the new distance between two nodes $\left(d_{(i j, t)}\right)$ is given by Eq. 9.

$$
d_{(i j, t)}=\sqrt{\left|X_{i}^{\prime}-X_{j}^{\prime}\right|^{2}+\left|Y_{i}^{\prime}-Y_{j}^{\prime}\right|^{2}} .
$$

2) The expiration time of the path: Let communication radius of the node is $R$, the expiration time of the link is $\operatorname{LET}(i, j)$, and the expiration time of the path is PET. $\operatorname{LET}(i, j)$ is given by Eq. 10 .

$$
\operatorname{LET}(i, j)=\frac{-(p q+u v)+\sqrt{\left(p^{2}+u^{2}\right) R^{2}+(p v-q u)^{2}}}{p^{2}+u^{2}} .
$$

Where $p=V_{i} \operatorname{Cos} \alpha-V_{j} \operatorname{Cos} \beta, q=X_{i}-X_{j}$, $u=V_{i} \operatorname{Sin} \alpha-V_{j} \operatorname{Sin} \beta, v=Y_{i}-Y_{j}$.

Since the whole path consists of $k$ links, the expiration time of the path is determined by the expiration time of each link. PET is given by Eq. 11.

$$
P E T=\min _{i \in(1,2, \ldots, k)}(\operatorname{LET}(i, j)) .
$$

\section{Performance ANAlysis of SNBR Protocol}

\section{A. Expected Path Lifetime of SNBR}

The stability of the path is determined by lifetime of the path. Therefore, we derive the Cumulative Distribution Functions (CDF) of the SNBR's path lifetime in this section.

The path from a source node $S$ to its destination node $D$ includes a primary path and its corresponding backup path. The two paths compose of disjoint sub-paths, and their common nodes are replaced by stable nodes. At the same time, length of the sub-paths is 3 hops. The primary path is the shortest distance path connected by stable nodes and intermediate nodes. The stable nodes split the whole path into $m-1$ sub-paths, the $i$ th sub-path is noted as $L_{i}(\mathrm{i}=1,2, \ldots$, $m-1$ ), and its corresponding lifetime is $X_{L_{i}} . L_{i}$ 's backup path is represented as $B P_{i}$, the corresponding links of $B P_{i}$ are represented as $B P_{i}^{1}, B P_{i}^{2}$ and $B P_{i}^{3}$ with lifetimes of $X_{B P_{i}}^{1}, X_{B P_{i}}^{2}$ and $X_{B P_{i}}^{3}$ respectively. The backup path is represented as $X_{B P_{i}}$, and $X_{B P_{i}}$ is given by Eq. 12 .

$$
X_{B P_{i}}=\min \left(X_{B P_{i}}^{1}, X_{B P_{i}}^{2}, X_{B P_{i}}^{3}\right) \text {. }
$$

When the primary path fails, the backup path will continue to transmit messages. $X_{L B R_{i}}$ is represented as the longest lifetime of $X_{L_{i}}$ and $X_{B P_{i}}$, it is given by Eq. 13 .

$$
X_{L B R_{i}}=\max \left(X_{L_{i}}, X_{B P_{i}}\right) \text {. }
$$

The path lifetime $T$ from a source node $S$ to its destination node $D$ is given by Eq. 14 .

$$
\begin{aligned}
T & =\min \left(\max \left(X_{L_{1}}, X_{B P_{1}}\right), \max \left(X_{L_{2}}, X_{B P_{2}}\right), \ldots,\right. \\
& =\min \left(X_{L B R_{1}}, X_{L B P_{2}}, X_{L B P_{m-1}}\right) .
\end{aligned}
$$

The CDF of the path lifetime $T$ is given by Eq. 15 .

$$
\begin{aligned}
F_{T}(t) & =P T \leq t]=P\left[\operatorname { m i n } \left(\max \left(X_{L_{1}}, X_{B P_{1}}\right), \max \left(X_{L_{2}}, X_{B P_{2}}\right), \ldots\right.\right. \\
& \left.=P\left[\max \left(X_{L_{m-1}}, X_{B_{m-1}}\right)\right) \leq t\right] \\
& =1-\prod_{i=1}^{m-1}\left(1-P\left[X_{L B R_{i}} \leq t\right]\right) \\
& =1-\prod_{i=1}^{m-1}\left(1-F_{X_{L B R_{i}}}(t)\right) .
\end{aligned}
$$

We assumed that $X_{L_{i}}$ is an independently and identically distributed random variable and its Probability Density Function (PDF) approximately equals to the exponential distribution. The PDF of is $X_{L B R_{i}}, f_{X_{L B R_{i}}}(t)=\lambda_{i} e^{-\lambda_{i} t}[13]$, and $\lambda_{i}=(m-1) / l . l$ is represented as the average lifetime of link.

The CDF of $X_{L_{i}}, X_{B R_{i}}$ and $X_{L B R_{i}}$ are given by Eqs.16, 17 and 18.

$$
\begin{gathered}
F_{X_{L_{i}}}(t)=1-e^{-\frac{1}{l} t} . \\
F_{X_{B P_{i}}}(t)=1-e^{-\frac{3}{l} t} \cdot \\
F_{X_{L B P_{i}}}(t)=\left(1-e^{-\frac{1}{l} t}\right)\left(1-e^{-\frac{3}{l} t}\right) .
\end{gathered}
$$

The CDF of the path lifetime $T$ is given by Eq. 19 .

$$
\begin{aligned}
F_{T}(t) & =1-\left[1-F_{X_{L_{i}}}(t) F_{X_{B P_{i}}}(t)\right]^{m-1} \\
& =1-\left[1-F_{X_{L B R_{i}}}(t)\right]^{m-1} \\
& =1-\left[1-\left(1-e^{-\frac{1}{l} t}\right)\left(1-e^{-\frac{3}{l} t}\right)\right]^{m-1} \\
& =1-\left(e^{-\frac{1}{l} t}+e^{-\frac{3}{l} t}-e^{-\frac{4}{l} t}\right)^{m-1}
\end{aligned}
$$




\section{B. Path Lifetime Comparison of SNBR and BSR Protocol}

In order to study the path lifetime of SNBR protocol, we conduct a comparison between SNBR and BSR protocol.

BSR is a multipath routing protocol and may have common nodes or links between the paths. Its cost function is achieved by the link similarity and the node similarity [7]. 20 [7].

The CDF of the path lifetime $T$ in BSR is given by Eq.

$$
F_{T}(t)=1-e^{-k t} \prod_{j=1}^{w}\left(e^{-h_{j} t}+e^{-h_{j}^{\prime} t}-e^{-h_{j} t-h_{j}^{\prime} t}\right) .
$$

Where $k$ is the number of the same links between the primary path and the backup path, $w$ is the number of disjoint sub-paths, $h_{j}$ and $h_{j}^{\prime}$ are the lengths of the corresponding disjoint sub-paths $(j=1,2, \ldots, w)$.

In order to verify the performance of SNBR protocol, we compare SNBR protocol with BSR protocol. The setting of the corresponding parameters is shown in Table 2.

TABLE II. THE CORRESPONDING PARAMETERS' SETTING FOR SBNR AND BSR PROTOCOL

\begin{tabular}{|c|c|}
\hline$l$ & $1 \mathrm{~s}$ \\
\hline$k$ & 0 \\
\hline$w=m-1$ & $1,2,3,4,5$ \\
\hline$h_{j}=h_{j}^{\prime}$ & 3 \\
\hline
\end{tabular}

In our analysis, as the length of the primary path changes in both SNBR and BSR protocol, we can achieve their corresponding expectation of path lifetime as shown in Figure 4.

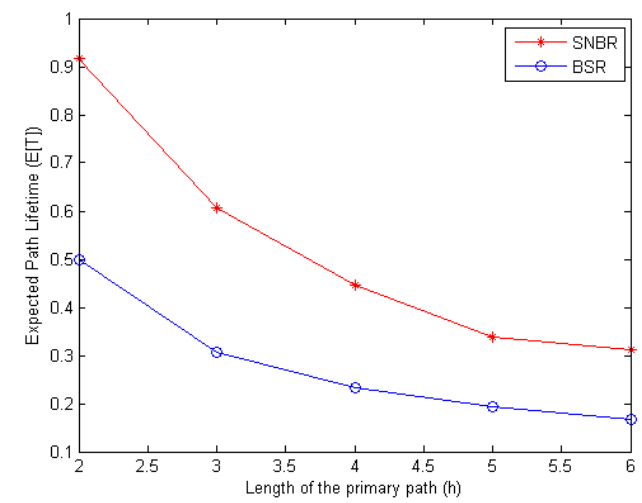

Figure 4. Expected path lifetime of different primary path length

Figure 4 shows that, with the increase of primary route length, the route lifetime is reduced. The reason is that with the increase of path length, the stability of the path is reduced. It is obvious that the lifetime expectancy of RNMR is significantly higher than BSR.

\section{CONCLUSION}

Topology dynamically changes which leads to links frequently interrupt, and thus it is hard to establish a stable path from source and destination. This paper presents a backup routing protocol based on the stable nodes (SNBR) to improve path stability in opportunistic networks. The path in SNBR composes of a primary path and a backup path, and their common nodes are replaced by the stable nodes. The stable nodes are determined by remaining power of the nodes, and the path between two stable nodes consists of disjoint sub-paths.

By comparing SNBR with BSR, it turns out that SNBR protocol has longer path lifetime than BSR. Therefore, the stability of the path in opportunistic networks is improved, and QoS of the network is also guaranteed at the same time.

\section{ACKNOWLEDGMENT}

This work was financially supported by the Foundation of Inner Mongolian Autonomous Region Educational Committee (NJZY11017), "Spring Sunshine Program" of the PRC Ministry of Education (z2009-1-01011), National Natural Science Foundation of China (61162006) and Research Program of Institutions of Higher Education of Inner Mongolia Autonomous Region (NJZZ12005).

\section{REFERENCES}

[1] Yongping Xiong, Limin Sun, Jianwei Niu, Yan Liu, "Opportunistic Networks," Journal of Software, vol.20, pp. 124-137, January 2009.

[2] Jorg Ott, Dirk Kutscher, Christoph Dwertmann, "Integrating DTN and MANET Routing," ACM, September 2006.

[3] Mina Masoudifar, "A review and performance comparison of QoS multicast routing protocols for MANETs," Ad Hoc Networks, pp. 1150-1155, 2009 .

[4] Charles E. Perkins, Elizabeth M. Royer, "Ad-hoc On-Demand Distance Vector Routing," IEEE, 2008.

[5] Mohammed Tarique, Kemal E. Tepe, Sasan Adibi and Shervin Erfani, "Survey of multipath routing protocols for mobile ad hoc networks," Journal of Network and Computer Applications, pp. 1125-1143, 2009.

[6] Xuefei Li and Laurie Cuthbert, "Node-Disjointness-Based Multipath Routing for Mobile Ad Hoc Networks,” ACM, pp. 23-29, 2004.

[7] Song Guo, Oliver Yang and Yantai Shu, "Improving Source Routing Reliability in Mobile Ad Hoc Networks," IEEE TRANSACTIONS ON PARALLEL AND DISTRIBUTED SYSTEMS, pp. 362-373, 2005.

[8] Mohammed Tarique, Kemal E. Tepe, Sasan Adibi and Shervin Erfani, "Survey of multipath routing protocols for mobile ad hoc networks," Journal of Network and Computer Applications, pp. 1125-1143, 2009.

[9] P.Rama Devi and D. Srinivasa Rao, "QOS ENHANCED HYBRID MULTIPATH ROUTING PROTOCOL FOR MOBILE AD HOC NETWORKS," International Journal of Distributed and Parallel Systems, 2012.

[10] LAURA MARIE FEENEY, "An Energy Consumption Model for Performance Analysis of Routing Protocols for Mobile Ad Hoc Networks," Mobile Networks and Applications, pp. 239-249, 2001.

[11] Nils Aschenbruck and Matthias Schwamborn, "Synthetic Map-based Mobility Traces for the Performance Evaluation in Opportunistic Networks," ACM, pp. 143-146, February 2010.

[12] Rajashekhar C. Biradar and Sunilkumar S. Manvi, "Ring Mesh Based Multicast Routing Scheme in MANET Using Bandwidth Delay Product," Wireless Pers Commun, pp. 117-146, 2012.

[13] S. Ross, "Introduction to Probability Models," Academic Press, 1989. 